HERIOT

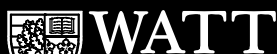

UNIVERSITY

Heriot-Watt University

Research Gateway

\title{
Integrated Front End Circuitry for Microultrasound Capsule Endoscopy
}

Citation for published version:

Lay, HS, Lemke, C, Cox, BF, Qiu, Y, Cummins, G, Ferrazzano, E, West, C, Desmulliez, MPY, Eddie Clutton, R \& Cochran, S 2019, Integrated Front End Circuitry for Microultrasound Capsule Endoscopy. in 2018 IEEE International Ultrasonics Symposium (IUS)., 8579738, IEEE International Ultrasonics Symposium (IUS), IEEE, 2018 IEEE International Ultrasonics Symposium, Kobe, Japan, 22/10/18. https://doi.org/10.1109/ULTSYM.2018.8579738

\section{Digital Object Identifier (DOI):}

10.1109/ULTSYM.2018.8579738

Link:

Link to publication record in Heriot-Watt Research Portal

Document Version:

Peer reviewed version

Published In:

2018 IEEE International Ultrasonics Symposium (IUS)

Publisher Rights Statement:

(C) 2019 IEEE. Personal use of this material is permitted. Permission from IEEE must be obtained for all other uses, in any current or future media, including reprinting/republishing this material for advertising or promotional purposes, creating new collective works, for resale or redistribution to servers or lists, or reuse of any copyrighted component of this work in other works.

\section{General rights}

Copyright for the publications made accessible via Heriot-Watt Research Portal is retained by the author(s) and / or other copyright owners and it is a condition of accessing these publications that users recognise and abide by the legal requirements associated with these rights.

\section{Take down policy}

Heriot-Watt University has made every reasonable effort to ensure that the content in Heriot-Watt Research Portal complies with UK legislation. If you believe that the public display of this file breaches copyright please contact open.access@hw.ac.uk providing details, and we will remove access to the work immediately and investigate your claim. 


\section{Integrated Front End Circuitry for Microultrasound Capsule Endoscopy}

\author{
Holly S Lay \\ Formerly University of Glasgow \\ Now FUJIFILM VisualSonics \\ Toronto, Canada \\ holly.lay@,fujifilm.com
}

\author{
Christina Lemke \\ School of Engineering \\ University of Glasgow \\ Glasgow, UK \\ christina.lemke@glasgow.ac.uk
}

Gerard Cummins School of Engineering and Physical Sciences

Heriot-Watt University

Edinburgh, UK

g.cummins@hw.ac.uk

\author{
Elena Ferrazzano \\ School of Engineering \\ University of Glasgow \\ Glasgow, UK
}

\author{
Benjamin F Cox \\ School of Medicine \\ University of Dundee \\ Dundee, UK \\ b.cox@dundee.ac.uk
}

R Eddie Clutton

The Royal (Dick) School of

Veterinary Studies

University of Edinburgh

Edinburgh, UK

e.clutton@ed.ac.uk

\begin{abstract}
Video capsule endoscopy (VCE) was originally developed to address the limitation of conventional endoscopy in accessing the small bowel as a remote part of the gastrointestinal tract. To further enhance the diagnostic ability of VCE, microultrasound capsule endoscopy is under development for identification of disease at an earlier stage and visualisation of subsurface tissue features. This paper presents an evaluation of two approaches to improve signal to noise ratio (SNR) in rapid prototyped capsule endoscopes. First, noise reduction techniques are applied to the integrated front-end circuits in the prototype capsules. Secondly, multiple types of coded excitation transmission are tested and benchmarked with respect to non-coded transmission. Results are presented for both bench top phantom imaging and in vivo translational trial imaging.
\end{abstract}

Keywords - capsule endoscopy, microultrasound, front-end electronics, coded excitation, pulse compression.

\section{INTRODUCTION}

Video capsule endoscopy (VCE) has now achieved technological maturity as an alternative to conventional endoscopy and colonoscopy but is limited to optical imaging of the gut wall surface for clinical diagnosis [1]. Research is now under way to expand this imaging platform with integrated ultrasound and microultrasound ( $\mu$ US) imaging devices (USCE) [2]-[4]. Researchers have particularly sought to address key concerns about acoustic coupling, image quality and multimodal system integration through a set of translational trial prototypes developed for rapid validation and design iteration [4]. Low-cost approaches with minimal manufacturing time were adopted to allow multiple rapid prototyping design cycles and integrated front-end electronics were included to improve signal integrity and address issues related to electrical interference. However, these low-cost approaches led to reduced performance and lower signal quality in comparison to clinical devices, in the raw echo data, resulting in images with reduced dynamic range and penetration depth.

This paper reports work to address the reduction of signal to noise ratio (SNR) while maintaining the form factor and rapid prototyping approach of the first devices. To reduce the overall noise floor of the system, analogue front-end circuitry was developed with improved isolation and reduced complexity. These circuits were then implemented in second generation USCE devices which were evaluated using the methodology adopted for and proven with the original capsules [4].

Simultaneously, coded excitation signals with suitable bandwidth and time-domain properties were identified and evaluated with a bench-top testing system [5] and in vivo testing to determine their suitability for use given the imaging window and power limitations of the capsule modality.

\section{FRONT-END ELECTRONICS}

\section{A. First Generation Electronics}

The design of the front-end electronic circuitry in early prototype capsules was intended to maximise the channel count while maintaining maximum acceptable outside dimensions (30 $\mathrm{mm}$ length, $10 \mathrm{~mm}$ diameter) based on existing VCE devices [6]. This was to allow transducers to be placed along the entire length of the capsule as well as at $180^{\circ}$ around the circumference of the capsule to assess the ability to establish acoustic coupling at different points along its length. The final design supported 
four single-element, physically focused polyvinylidene fluoride (PVDF) transducers (30 MHz centre frequency, $6 \mathrm{~mm}$ focal depth) with onboard transmit/receive isolation (MD0101, Microchip Technology, Chandler, USA) and $12 \mathrm{~dB}$ amplification achieved through commercial operational amplifiers (ADA4807, Analog Devices, Norwood, USA). These were mounted on a custom printed circuit board (PCB, Eurocircuits N.V., Mechelen, Belgium), with the transducer wires directly attached via through-hole connections. A tether was used for power supply and data communication, with connectivity to the circuitry within the USCE device achieved with individual micro-coaxial cables (42 AWG core, 9442 WH033, AlphaWire, Elizabeth, USA).

\section{B. Second Generation Electronics}

While good transducer to tissue coupling was achieved with the described design and acceptable M-mode images were produced using a pull-back approach, the high-density prototype proved prone to electrical noise and oscillation in the amplifiers. The transmit/receive $(\mathrm{T} / \mathrm{R})$ protection circuits also did not recover well from avalanche mode transmit signals. As the first set of in vivo trials proved the imaging capability of the capsule was relatively independent of transducer position, it was judged reasonable to reduce the number of transducers from four to two, allowing a significant reduction in circuit density.

The reduced channel count allowed the transmit and receive traces to be routed with more direct connections to the circuitry. This also provided space for the placement of electrical ground planes between all traces while removing them from under the sensitive amplifier chip. The micro-coaxial shields were isolated from the board ground plane and tied together at a single point at the proximal end of the tether to avoid current loops. Additionally, the $\mathrm{T} / \mathrm{R}$ chips were replaced with twin diode and capacitor protection circuits [7] (BAV99, Vishay Intertechnology, Inc., Malvern, USA). These changes resulted in a much lower circuit density (Fig. 1), simplifying manufacturing and decreasing cross-talk. The redesign was also achieved within the same footprint as the original PCB, minimising the capsule shell redesign.

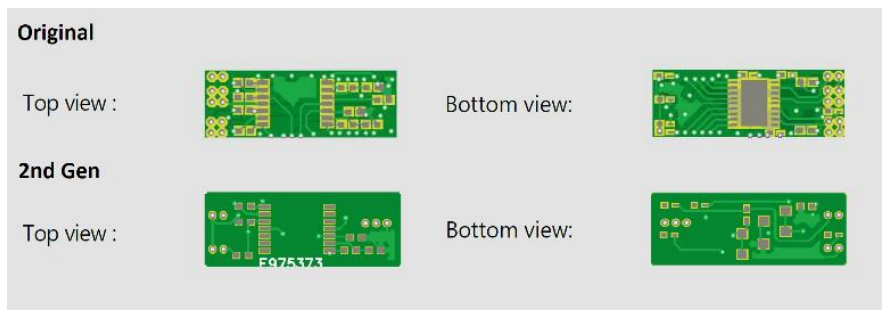

Fig. 1. Comparison of printed circuit board design for the 1st generation (top) and 2nd generation (bottom) USCE device. All boards measured $18 \times 6.3 \mathrm{~mm}$.

\section{CODED EXCITATION}

Autonomous USCE capsules have to rely on a power supply with a limited maximum voltage. This voltage can be increased with charge-pump based power-management but these have limited conversion efficiency. This in turn suggests that techniques able to operate with lower maximum voltage will allow enhanced SNR and penetration depth, compatible with
USCE operating lifetime. One approach explored is the use of coded excitation signals, which have found applications in medical ultrasound [8] and non-destructive testing [9] but have seen only limited use in $\mu$ US [10].

\section{A. Background}

In contrast to a conventional broadband pulse, coded excitation waveforms are frequency modulated (chirps), phaseencoded (Barker code, Golay codes) or time interval encoded [11]. They are normally subject to decoding on receive to recover the higher resolution of a shorter pulse. Overall, an SNR gain of $20 \mathrm{~dB}$ is thought to be realistic in soft-tissue imaging [12]. Optionally, mismatched filtering, e.g. by addition of a Dolph-Chebychev window, may be applied to smooth the results at a cost of a few dB SNR [13].

The transducer frequency response and imaging medium both affect the quality of coded excitation as illustrated in Fig. 2 and should be taken into account when selecting waveforms for a particular application [14]. This is especially relevant in tissue as it is inhomogeneous and has significant non-linear properties.

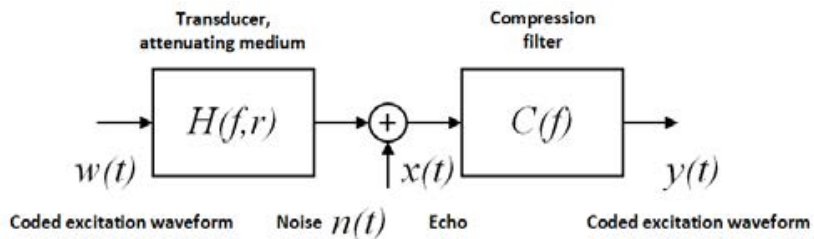

Fig. 2. System model of a coded excitation ultrasound system, after [14], with $H(f, r)$ the transfer function of the tranducer and the medium with frequency and depth $r$ dependent attenuation, $n(t)$ additive noise and compression filter $C(f)$.

\section{B. Waveform Selection}

Our goal was to identify suitable waveforms for use in benchtop tests and in-vivo trials. Linear frequency-modulated (FM) chirps are good candidates as they have smooth second derivatives which are less prone to distortion through the transducer. Barker codes and Golay code pairs were also considered, as they have the minimum sidelobe level of single transmission codes and no sidelobes, respectively.

To match the physical parameters of the PVDF transducers in the USCE test devices, the frequency range of the chirps was defined as $10-45 \mathrm{MHz}$ and the binary phase-encoded Barker and Golay codes were modulated with $30 \mathrm{MHz}$ sinusoidal signals. The waveforms were further tuned to accommodate the characteristic output of the bespoke power amplifier in the experimental setup. Consequently, the chirps were multiplied with a tapered cosine (Tukey) window instead of a Hanning window to maximise signal consistency and energy. The selected Barker and Golay codes had multiple cycles rather than one cycle per bit and a pause between subsequent bits to preserve the phase changes post-amplification, Fig. 3.

1. The coded waveforms finally selected for in-vivo testing were $10-45 \mathrm{MHz}$ linear FM chirps with lengths of $353 \mathrm{~ns}, 919$ ns and 1767 ns, tapered with 75\% Tukey windows. Furthermore, a 13 bit Barker code, and 4 bit and 8 bit Golay code pairs were 

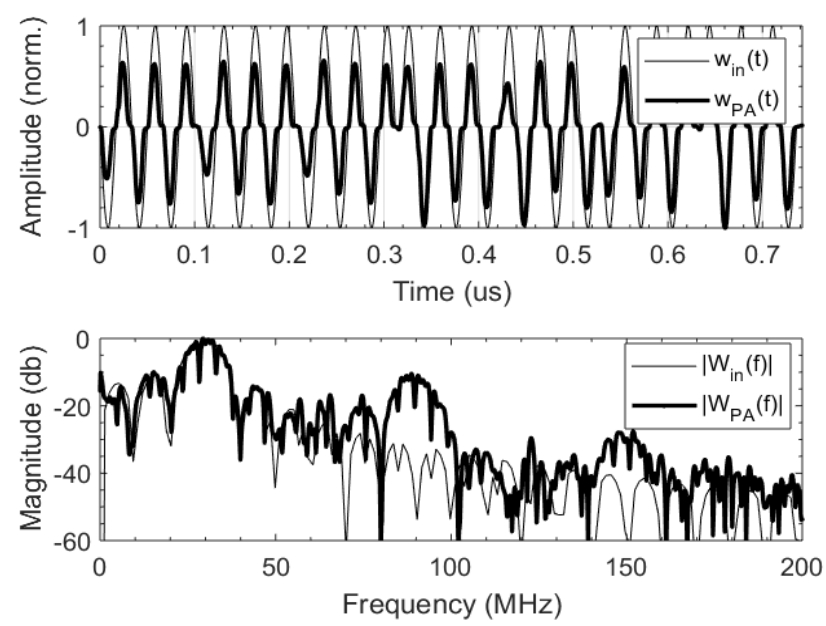

Fig. 3. Example of a 7 bit Barker coded waveform, modulated with a $30 \mathrm{MHz}$ sine. The code $w(t)$ is designed with three harmonic cycles per bit and a pause between each bit of $2 \mathrm{~ns}$. The thick line $(\mathrm{hTx}(\mathrm{t}))$ shows the the signal at the power amplifier output with amplitude distortion, but preserved phase changes.

chosen, each modulated with three full cycles of a sinusoid per bit and a pause of $2 \mathrm{~ns}$ between consecutive bits.

\section{Scanning System Tests}

Test images were generated with the selected waveforms using a static scanning system and a $25 \mu \mathrm{m}$ tungsten wire target. An arbitrary waveform generator (4162, Rigol Technologies, Inc, Suzhou, China) was used to generate the codes, which were input to the bespoke amplifier to drive a $30 \mathrm{MHz}$ PVDF single element transducer with $6.5 \mathrm{~mm}$ spherical focal length.

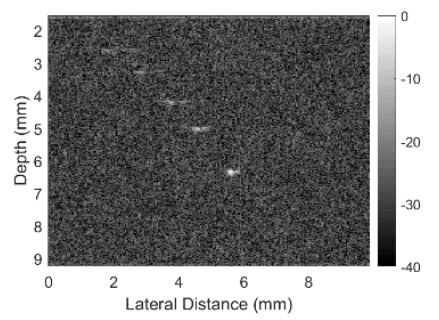

(a)

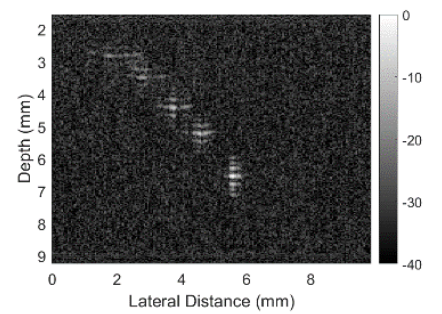

(c)

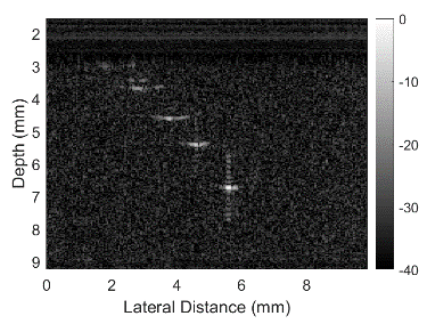

(b)

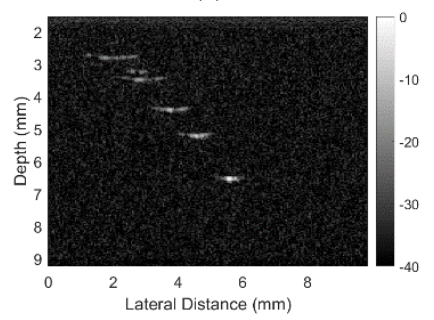

(d)
Fig. 4. Wire target scan results with (a) 353ns chirp, (b) 13 bit Barker code, (c) 8 bit Golay Code (Part A of the code pair only), (d) 8 bit Golay Code (postcode pair summation).

Fig. 4 shows an example of the scan results. As expected, the best resolution in all scans was obtained at the physical focus of the transducer. The 353 ns chirp image shows visible wire echoes but the dynamic range is less than the other codes due to the shorter pulse length and corresponding lower pulse energy. The 13 bit Barker code and the 8 bit Golay code part A show their characteristic sidelobes and an increased dynamic range. The best result with regards to dynamic range and resolution is achieved with the combined 8 bit Golay code.

\section{TRANSLATION TRIAL VALIDATION}

Validation of the new prototype electronics was performed in the small bowel of a porcine model in vivo. The procedure was conducted under Home Office (UK) License PPL:70/8812 in accordance with the Animal (Scientific Procedures) Act 1986.

Two female Landrace pigs, aged 4 months, were supplied by a local breeder/supplier. The pigs were maintained in groups of no less than two animals in licensed housing (PEL:60/4604), bedded on straw and fed 'ABN Pig Rearer Pellets' ad lib. Environmental variables were maintained within the limits detailed by PEL. Food was withheld for 12 hours before anesthesia but access to water was maintained until preanesthetic medication which comprised: azaperone (1 mgkg-1; "Stresnil $40 \mathrm{mg} / \mathrm{ml}$ Solution for Injection", Elanco Animal Health, Hampshire), ketamine (2 mgkg-1; "Ketamidor 100 $\mathrm{mg} / \mathrm{ml}$ Solution for Injection", Chanelle UK, Berkshire), midazolam (0.5 mgkg-1; "Hypnovel $10 \mathrm{mg} / 2 \mathrm{ml}$ Solution for Injection", Roche, Hertfordshire) and morphine (0.25 mgkg-1; "Morphine Sulphate $30 \mathrm{mg} / \mathrm{ml}$ ", Martindale Pharmaceuticals, Buckinghamshire) combined in one syringe and injected intramuscularly. Anesthesia was induced with isoflurane ("Isoflo" Zoetis, Surrey), vaporized in oxygen and nitrous oxide administered via a Bain breathing system and facemask. A cannula was placed in the auricular vein.

Access to the remote small bowel was achieved via an artificially created stoma under general anesthesia immediately prior to the experiment. The experiment and subject were monitored throughout the procedure by an experienced veterinary anesthetist. When the experiments were complete, the pigs were euthanized without recovery using pentobarbital.

The USCE devices were introduced $\sim 30 \mathrm{~cm}$ into the small bowel via the stoma and M-mode scans were obtained for static positions and with manual pull-back of the devices by the clinician. All pullbacks were performed over $30 \mathrm{~s}$ intervals with an $8 \mathrm{~cm}$ pull length. After each pullback, the capsule was returned to its original position by means of measured marks on the tether. All scans were reconstructed using standard envelope detection and logarithmic compression and evaluated by the clinician for image quality and ability to discern target anatomy.

\section{RESUlTS, Discussion AND CONCLUSIONS}

The second-generation front-end circuitry performed well in translational trials, with improved tissue differentiation in comparison to the first generation, as shown in Fig. 5. Refinement of the pullback procedure based on experience gained from preceding trials also led to improved scan repeatability over multiple pullbacks. Overall, the imaging clinician believed the results to be satisfactory and confirmed that they represented an improvement over previous trial 


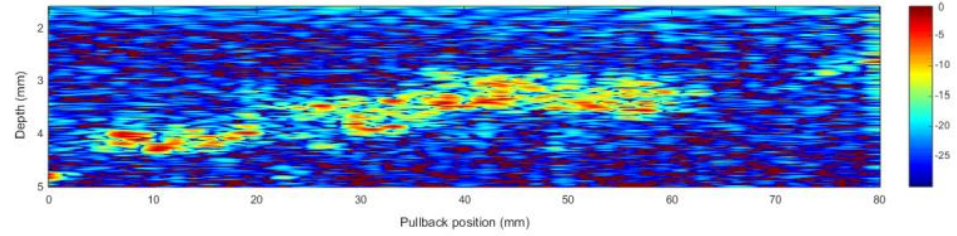

(a)

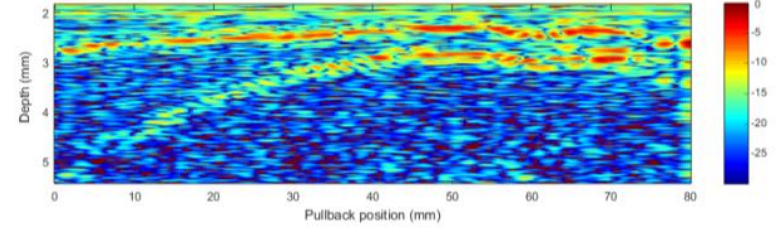

(b)

Fig. 5. Comparison of image quality for in vivo M-mode pullbacks using (a) first generation front-end circuitry and (b) second generation circuitry. Direct comparison is impossible because the measurements were taken in two separate experiments with different pigs but enhanced tissue imaging and penetration is apparent in (b).

outcomes. In contrast, theresults achieved with the selected coded waveforms showed limited visibility in the underlying tissue structures.

The reduced performance with coded excitation in vivo may be caused by increased sensitivity to tissue motion and nonlinear effects compared to conventional imaging modalities. Additionally, the bespoke amplifier used with the coded excitation configuration had lower peak power than the standard imaging system. However, the experiments successfully demonstrated that both front-end circuit designs were compatible with the coded excitation system and the particular codes selected. Future waveform design will explore ways to address the non-linearity and motion sensitivity of the waveforms tested in this study.

\section{REFERENCES}

[1] G. Ciuti, A. Menciassi, and P. Dario, "Capsule Endoscopy: From Current Achievements to Open Challenges," IEEE Rev. Biomed. Eng., vol. 4, pp. 59-72, 2011.

[2] F. Memon et al., "Capsule ultrasound device: Further developments," in 2016 IEEE International Ultrasonics Symposium (IUS), 2016, pp. 1-

[3] J. Wang et al., "Capsule Ultrasound Device: Characterization and Testing Results," in Proceedings 2017 IEEE International Ultrasonics Symposium, 2017, pp. 1-4.

[4] H. Lay et al., "In-Vivo Evaluation of Microultrasound and Thermometric Capsule Endoscopes," IEEE Trans. Biomed. Eng., Jul. 2018.

[5] H. S. Lay et al., "Microultrasound characterisation of ex vivo porcine tissue for ultrasound capsule endoscopy," J. Phys. Conf. Ser., vol. 797, p. 012003, Jan. 2017.
[6] A. Sieg, "Capsule endoscopy compared with conventional colonoscopy for detection of colorectal neoplasms," World J. Gastrointest. Endosc., vol. 3, no. 5, pp. 81-85, May 2011.

[7] G. R. Lockwood, J. W. Hunt, and F. S. Foster, "The design of protection circuitry for high-frequency ultrasound imaging systems," IEEE Trans. Ultrason. Ferroelectr. Freq. Control, vol. 38, no. 1, pp. 48-55, Jan. 1991.

[8] T. Misaridis and J. A. Jensen, "Use of modulated excitation signals in medical ultrasound. Part I: basic concepts and expected benefits," IEEE Trans. Ultrason. Ferroelectr. Freq. Control, vol. 52, no. 2, pp. $177-$ 191, Feb. 2005.

[9] R. Su et al., "Coded Excitation for Air-Coupled Ultrasound Phased Arrays," 56th Annu. Conf. Br. Inst. Non-Destr. Test. Proc., pp. 250257, 2017.

[10] W. Qiu et al., "Modulated Excitation Imaging System for Intravascular Ultrasound," IEEE Trans. Biomed. Eng., vol. 64, no. 8, pp. 1935-1942, Aug. 2017.

[11] J. Isla and F. Cegla, "Coded Excitation for Pulse-Echo Systems," IEEE Trans. Ultrason. Ferroelectr. Freq. Control, vol. 64, no. 4, pp. 736748, Apr. 2017.

[12] M. O'Donnell, "Coded excitation system for improving the penetration of real-time phased-array imaging systems," IEEE Trans. Ultrason. Ferroelectr. Freq. Control, vol. 39, no. 3, pp. 341-351, 1992.

[13] A. Misaridis, "Ultrasound imaging using coded signals," Technical University of Denmark, Department of Electrical Engineering, 2001.

[14] A. R. Brenner, K. Eck, W. Wilhelm, and T. G. Noll, "Improved resolution and dynamic range in medical ultrasonic imaging using depth-dependent mismatched filtering," in 1997 IEEE Ultrasonics Symposium Proceedings. An International Symposium (Cat. No.97CH36118), 1997, vol. 2, pp. 1475-1480 vol.2.

This work was supported by the UK Engineering and Physical Sciences Research Council (EPSRC) under the grant number EP/K034537/2, "Sonopill". 